\title{
Influence on attitudes and lifestyle due to lockdown amidst COVID-19 pandemic: a perception-based analysis among Bangladeshi residents
}

Md. Saiful Islam ${ }^{1,2+}$, Md. Asad Ullah ${ }^{3 \dagger}$, Ummay Soumayia Islam', Sahadat Hossain ${ }^{1}$, Yusha Araf ${ }^{4}$, Anindya Das ${ }^{5}$, Asir Newaz Khan ${ }^{6}$, Nafisa Nawal Islam³ ${ }^{3}$ Bishajit Sarkar ${ }^{3}$ and Abdullah Mohammad Shohael ${ }^{3^{*}}$

\begin{abstract}
Background: Countrywide lockdown or stay-at-home order has been implemented to slow down the transmission of emergent coronavirus. However, the influence on attitudes and lifestyle due to lockdown amidst the coronavirus disease 2019 (COVID-19) pandemic has been poorly understood. The present study aimed to investigate the influence on attitudes and lifestyle due to lockdown amidst the COVID-19 pandemic among Bangladeshi residents.

Methods: A cross-sectional survey carried out involving 1635 community dwellers across eight divisions in Bangladesh conducted from April 15, 2020 to May 10, 2020. A structured questionnaire incorporating sociodemographic, attitudes towards lockdown and adverse lifestyle amidst lockdown measures was employed to collect data using the Google Forms. Multiple regression analyses were executed to determine the associated factors of positive attitudes towards lockdown and adverse lifestyle.

Results: The mean scores of attitudes towards lockdown were $67.9(S D=8.4)$ out of 85 with an overall correct rate (positive attitudes) of 79.9\%; whereas the mean scores of adverse lifestyle amidst lockdown were 16.1 (SD = 4.8) out of 34 with an overall rate of $47.4 \%$. The factors associated with more positive attitudes towards lockdown included being female, divorced, higher educated, and students. Conversely, being male, having no formal education, and rural residence were associated factors of adverse lifestyle amidst the COVID-19 pandemic.

Conclusions: The findings reflect how the COVID-19 lockdown has preciously impacted the attitudes, and lifestyle of Bangladeshi citizens, which will contribute to promoting appropriate measures during a subsequent zonal or complete lockdown.
\end{abstract}

Keywords: COVID-19, Lockdown, Attitude, Lifestyle, Bangladesh

\footnotetext{
* Correspondence: amshohael@juniv.edu

${ }^{+}$Md. Saiful Islam and Md. Asad Ullah contributed equally to this work.

${ }^{3}$ Department of Biotechnology and Genetic Engineering, Jahangirnagar University, Savar, Dhaka, Bangladesh

Full list of author information is available at the end of the article
}

(c) The Author(s). 2021 Open Access This article is licensed under a Creative Commons Attribution 4.0 International License, which permits use, sharing, adaptation, distribution and reproduction in any medium or format, as long as you give appropriate credit to the original author(s) and the source, provide a link to the Creative Commons licence, and indicate if changes were made. The images or other third party material in this article are included in the article's Creative Commons licence, unless indicated otherwise in a credit line to the material. If material is not included in the article's Creative Commons licence and your intended use is not permitted by statutory regulation or exceeds the permitted use, you will need to obtain permission directly from the copyright holder. To view a copy of this licence, visit http://creativecommons.org/licenses/by/4.0/ The Creative Commons Public Domain Dedication waiver (http://creativecommons.org/publicdomain/zero/1.0/) applies to the data made available in this article, unless otherwise stated in a credit line to the data. 


\section{Background}

The novel coronavirus that causes a severe respiratory disease also known as COVID-19 was first reported by the World Health Organization (WHO) on December 31, 2019 and got recognition as a global pandemic on March 11, 2020 [1, 2]. Since the first outbreak of the disease in Wuhan, China, the infectious virus started its ravaging effect all over the world except Antarctica [35]. The major symptoms of COVID-19 include fever, dry cough, fatigue, myalgia, breathing difficulty and dyspnea [6-8]. Lungs are the most affected organs as the virus enters through the angiotensin converting enzyme 2 that are mostly prolific in the type 2 alveolar cells of the lungs [9]. Due to lack of sufficient knowledge about the disease, health care systems from third to first world countries struggled to treat the massive number of infected COVID-19 patients [10]. Since COVID-19 disseminates through social contact and there was no medicine available to cure the disease, billions of people all over the world went under lockdown measures to minimize the transmission rate $[11,12]$. The active involvement of every person on earth with respect to testing, isolation, contact tracing, social distancing, home quarantine, self-quarantine, maintaining hygiene and using personal protective equipment has become important to combat COVID-19's impact as well as give sufficient time for developing treatment strategies [13, 14]. However, due to varying level of knowledge, attitudes and practices towards the COVID-19 pandemic prevention strategies has brought challenges in many countries $[10,15]$.

The Institute of Epidemiology, Disease Control and Research (IEDCR, the national institute for conducting disease surveillance and outbreak investigation in Bangladesh) confirmed the country's first cases of coronavirus on March 8, 2020 [16, 17], and since then to safeguard the citizens, the government of Bangladesh declared a countrywide lockdown from March 26, to May 31, 2020 [18-20]. As of November 20, 2020 Bangladesh confirmed 4,41,159 cases with 6305 deaths and 3,56,722 recoveries [21]. Meanwhile, similar partial or complete lockdown measures were created in other countries; they have disrupted the daily routines of almost two-thirds of the world's population. Since the lockdown enforcement imposes restrictions on people's rights by refraining them from normal day-to-day activities, its usefulness remains a subject of debate among different groups of people, as evidenced by various poll studies during this pandemic [22]. Thus, it will be useful and timely to understand the specific public perception, activities, and attitudes during the lockdown so governments and policymakers can regulate, recommend, and take necessary steps to avoid any undesired outcome and fulfill their basic needs [23]. In addition, this lockdown has affected people of Bangladesh based on their knowledge, attitude, and practices; those who are older, more educated, employed, have a monthly family income over 30,000 BDT (Bangladeshi Taka), and reside in urban areas showed more positive attitude towards the COVID-19 pandemic [24]. Due to the restrictions of normal routine life activities during the complete countrywide lockdown the public's perception, attitude, and activities erupted, and therefore, it is crucial to understand their response for implementing further steps to alleviate their grievances in the long run given the uncertainty surrounding the pandemic which is in the process of being under control.

To date, there are very limited studies that have been executed in Bangladesh regarding people's attitudes or perceptions across socio-demographic conditions during a countrywide lockdown [24, 25]. In this paper, we quantitatively examined how the people of Bangladesh from different socio-economic status (SES) responded including their daily activities, physical and mental conditions during the countrywide lockdown, which in turn impacted the overall effectiveness of this restrictive measure. The present study was the first report on COVID-19, which covers the people living in both urban and rural areas of all divisions in Bangladesh, capturing the holistic dimension of the influence of attitudes and lifestyle due to lockdown of community people. Thus, the present study aimed to investigate the influence on attitudes and lifestyle due to lockdown amidst the COVID-19 pandemic among Bangladeshi residents. The scientific findings of this study will contribute to recommend necessary and emergency steps that are required to avoid any perilous situation during any further zonal or complete countrywide lockdown in the future.

\section{Methods \\ Study design and participants}

This was a cross-sectional survey conducted among 1635 community dwellers across eight divisions (i.e., Dhaka, Chittagong, Rajshahi, Khulna, Barishal, Sylhet, Rangpur, and Mymensingh) of Bangladesh covering both urban and rural areas. Data were collected from April 15 , to May 10, 2020, when the country was under complete lockdown, which was enforced on March 26, 2020. The study's target population was Bangladeshi citizens who were house-bound in Bangladesh during the COVID-19 pandemic. The inclusion criteria included being (i) aged 18 years or older; (ii) able to understand Bangla, and (iii) able to complete the entire survey.

\section{Sample size calculation}

The sample size was calculated using the following Eq. (1): 


$$
\begin{aligned}
& n=\frac{z^{2} p q}{d^{2}} \\
& \Rightarrow n=\frac{1.96^{2} \times 0.5 \times(1-0.5)}{0.05^{2}} \\
& \Rightarrow n=384.16 \approx 384
\end{aligned}
$$

Here,

$n=$ number of samples

$z=1.96$ (95\% confidence level)

$p=$ prevalence estimate $(50 \%$ or 0.5$)$ (as no study found)

$$
\begin{aligned}
& q=(1-p) \\
& d=\text { precision limit or proportion of sampling error }
\end{aligned}
$$
(0.05)

As there is no prior similar study focusing on the influence on attitudes and lifestyle due to lockdown amidst the COVID-19 pandemic in Bangladesh, we made the best assumption (p) for the present study would be $50 \%$. Assuming a $10 \%$ non-response rate, a sample size of $423.5 \approx 424$ participants was estimated. Our sample size exceeded this estimate.

\section{Data collection procedures}

A self-reported questionnaire incorporating in the Google Forms was employed to survey participants. A total of 24 research assistants who completed their graduation in health and life sciences along with clinicians and statisticians approached the participants to take part in the survey. The age-stratified (over 18 years) convenient sampling method utilized to recruit the participants. In sum, 1635 participants took part in the survey prior to informed consents.

\section{Measures}

A structured questionnaire incorporating three sections (i.e., socio-demographic information, attitudes towards lockdown, and adverse lifestyle amidst lockdown measures - see below) was utilized to collect data during the data collection periods. At first, a draft questionnaire was prepared based on extensive literature review [2631].Then it was piloted among 20 individuals in an urban slum of Dhaka city, and then the questionnaire was finalized after checking of its validity by the academic experts knowledgeable in this area. These pilot data were excluded from the final analysis.

\section{Socio-demographic information}

Some basic information of participants was recorded during the survey including age (later categorized: 1825 years and over 25 years), gender (male/female), Religion (Muslim/Hindu/Buddhist/Christian), marital status (unmarried/married/divorced), and residence (rural/ urban). In addition, participants' education, occupation and resident division were also asked.

\section{Attitudes towards lockdown measures}

The attitudes towards lockdown were measured using

\begin{tabular}{|c|c|c|}
\hline Characteristics & $n$ & $(\%)$ \\
\hline \multicolumn{3}{|l|}{ Gender } \\
\hline Male & 1005 & $(61.5)$ \\
\hline Female & 630 & (38.5) \\
\hline \multicolumn{3}{|l|}{ Age } \\
\hline Young (18-25 years) & 1015 & $(62.1)$ \\
\hline Older (> 25 years) & 620 & $(37.9)$ \\
\hline \multicolumn{3}{|l|}{ Religion } \\
\hline Muslim & 1381 & $(84.5)$ \\
\hline Hindu & 240 & $(14.7)$ \\
\hline Buddhist & 14 & $(0.9)$ \\
\hline \multicolumn{3}{|l|}{ Marital status } \\
\hline Unmarried & 1002 & $(61.3)$ \\
\hline Married & 619 & $(37.9)$ \\
\hline Divorced & 14 & $(0.9)$ \\
\hline \multicolumn{3}{|l|}{ Education } \\
\hline No formal education & 59 & (3.6) \\
\hline Primary & 67 & $(4.1)$ \\
\hline Secondary & 180 & $(11.0)$ \\
\hline Higher Secondary & 1043 & $(63.8)$ \\
\hline University & 253 & $(15.5)$ \\
\hline Higher education & 33 & $(2.0)$ \\
\hline \multicolumn{3}{|l|}{ Occupation } \\
\hline Student & 936 & $(57.2)$ \\
\hline Housewife & 191 & $(11.7)$ \\
\hline Service Holder (Govt.) & 226 & (13.8) \\
\hline Business & 145 & (8.9) \\
\hline Unemployed/other & 137 & $(8.4)$ \\
\hline \multicolumn{3}{|l|}{ Residence } \\
\hline Rural & 670 & $(41.0)$ \\
\hline Urban & 965 & $(59.0)$ \\
\hline \multicolumn{3}{|l|}{ Resident division } \\
\hline Dhaka & 532 & $(32.5)$ \\
\hline Chittagong & 384 & $(23.5)$ \\
\hline Rajshahi & 86 & (5.3) \\
\hline Khulna & 322 & (19.7) \\
\hline Barishal & 89 & (5.4) \\
\hline Sylhet & 37 & $(2.3)$ \\
\hline Rangpur & 32 & (2.0) \\
\hline Mymensingh & 153 & (9.4) \\
\hline
\end{tabular}
structured questions based on the WHO and the Centers for Disease Control and Prevention (CDC) guidelines [26-31]. This section consisted of 17-item

Table 1 General characteristics of participants ( $N=1635)$ 
Table 2 Attitudes towards lockdown and gender difference of participants

\begin{tabular}{|c|c|c|c|c|c|c|c|c|c|}
\hline \multirow[t]{2}{*}{ Characteristics } & \multicolumn{2}{|c|}{ Total $N=1635$} & \multicolumn{2}{|c|}{ Male } & \multicolumn{2}{|c|}{ Female } & \multirow[t]{2}{*}{$x^{2}$} & \multirow[t]{2}{*}{$d f$} & \multirow[t]{2}{*}{$P$-value } \\
\hline & $n$ & (\%) & $n$ & (\%) & $n$ & (\%) & & & \\
\hline \multicolumn{10}{|c|}{ The government should provide food and necessary equipment to the unprivileged people } \\
\hline Strongly agree & 1196 & $(73.1)$ & 723 & $(71.9)$ & 473 & $(75.1)$ & 15.35 & 4 & .004 \\
\hline Agree & 207 & $(12.7)$ & 115 & $(11.4)$ & 92 & $(14.6)$ & & & \\
\hline Neutral & 109 & $(6.7)$ & 82 & $(8.2)$ & 27 & $(4.3)$ & & & \\
\hline Disagree & 44 & $(2.7)$ & 30 & (3.0) & 14 & $(2.2)$ & & & \\
\hline Strongly disagree & 79 & $(4.8)$ & 55 & $(5.5)$ & 24 & (3.8) & & & \\
\hline \multicolumn{10}{|c|}{ Lockdown can prevent community spreading } \\
\hline Strongly agree & 393 & $(24.0)$ & 248 & $(24.7)$ & 145 & $(23.0)$ & 11.66 & 4 & .020 \\
\hline Agree & 314 & $(19.2)$ & 193 & $(19.2)$ & 121 & $(19.2)$ & & & \\
\hline Neutral & 230 & $(14.1)$ & 160 & $(15.9)$ & 70 & $(11.1)$ & & & \\
\hline Disagree & 329 & $(20.1)$ & 197 & $(19.6)$ & 132 & $(21.0)$ & & & \\
\hline Strongly disagree & 369 & $(22.6)$ & 207 & $(20.6)$ & 162 & $(25.7)$ & & & \\
\hline \multicolumn{10}{|c|}{ Lockdown can increase crime in the city } \\
\hline Strongly agree & 284 & $(17.4)$ & 169 & $(16.8)$ & 115 & $(18.3)$ & 13.77 & 4 & .008 \\
\hline Agree & 361 & $(22.1)$ & 224 & $(22.3)$ & 137 & $(21.7)$ & & & \\
\hline Neutral & 293 & $(17.9)$ & 196 & $(19.5)$ & 97 & $(15.4)$ & & & \\
\hline Disagree & 238 & $(14.6)$ & 160 & $(15.9)$ & 78 & $(12.4)$ & & & \\
\hline Strongly disagree & 459 & $(28.1)$ & 256 & $(25.5)$ & 203 & $(32.2)$ & & & \\
\hline \multicolumn{10}{|c|}{ Lockdown will make the environment less polluted than ever } \\
\hline Strongly agree & 1111 & $(68.0)$ & 695 & $(69.2)$ & 416 & $(66.0)$ & 15.66 & 4 & .004 \\
\hline Agree & 326 & $(19.9)$ & 176 & $(17.5)$ & 150 & $(23.8)$ & & & \\
\hline Neutral & 108 & $(6.6)$ & 80 & (8.0) & 28 & $(4.4)$ & & & \\
\hline Disagree & 36 & $(2.2)$ & 21 & $(2.1)$ & 15 & $(2.4)$ & & & \\
\hline Strongly disagree & 54 & (3.3) & 33 & (3.3) & 21 & (3.3) & & & \\
\hline \multicolumn{10}{|c|}{ Lockdown can be the reason for various animal death for want of food } \\
\hline Strongly agree & 677 & $(41.4)$ & 391 & $(38.9)$ & 286 & $(45.4)$ & 13.19 & 4 & .010 \\
\hline Agree & 488 & $(29.8)$ & 294 & $(29.3)$ & 194 & (30.8) & & & \\
\hline Neutral & 251 & $(15.4)$ & 172 & $(17.1)$ & 79 & $(12.5)$ & & & \\
\hline Disagree & 137 & (8.4) & 91 & $(9.1)$ & 46 & $(7.3)$ & & & \\
\hline Strongly disagree & 82 & $(5.0)$ & 57 & $(5.7)$ & 25 & $(4.0)$ & & & \\
\hline \multicolumn{10}{|c|}{ Lockdown will create all sorts of medical therapy problems } \\
\hline Strongly agree & 436 & $(26.7)$ & 265 & $(26.4)$ & 171 & $(27.1)$ & 10.81 & 4 & .029 \\
\hline Agree & 645 & $(39.4)$ & 375 & $(37.3)$ & 270 & $(42.9)$ & & & \\
\hline Neutral & 358 & $(21.9)$ & 238 & $(23.7)$ & 120 & $(19.0)$ & & & \\
\hline Disagree & 126 & $(7.7)$ & 76 & $(7.6)$ & 50 & $(7.9)$ & & & \\
\hline Strongly disagree & 70 & $(4.3)$ & 51 & $(5.1)$ & 19 & $(3.0)$ & & & \\
\hline \multicolumn{10}{|c|}{ Lockdown will create workout problems } \\
\hline Strongly agree & 828 & $(50.6)$ & 498 & $(49.6)$ & 330 & $(52.4)$ & 5.42 & 4 & .247 \\
\hline Agree & 485 & $(29.7)$ & 291 & $(29.0)$ & 194 & $(30.8)$ & & & \\
\hline Neutral & 188 & $(11.5)$ & 127 & $(12.6)$ & 61 & $(9.7)$ & & & \\
\hline Disagree & 82 & $(5.0)$ & 55 & $(5.5)$ & 27 & $(4.3)$ & & & \\
\hline Strongly disagree & 52 & $(3.2)$ & 34 & (3.4) & 18 & $(2.9)$ & & & \\
\hline
\end{tabular}


Table 2 Attitudes towards lockdown and gender difference of participants (Continued)

\begin{tabular}{|c|c|c|c|c|c|c|c|c|c|}
\hline \multirow[t]{2}{*}{ Characteristics } & \multicolumn{2}{|c|}{ Total $N=1635$} & \multicolumn{2}{|c|}{ Male } & \multicolumn{2}{|c|}{ Female } & \multirow[t]{2}{*}{$x^{2}$} & \multirow[t]{2}{*}{$d f$} & \multirow[t]{2}{*}{$P$-value } \\
\hline & $n$ & (\%) & $n$ & (\%) & $n$ & (\%) & & & \\
\hline Strongly agree & 466 & $(28.5)$ & 277 & $(27.6)$ & 189 & $(30.0)$ & 18.12 & 4 & .001 \\
\hline Agree & 562 & $(34.4)$ & 338 & (33.6) & 224 & $(35.6)$ & & & \\
\hline Neutral & 353 & (21.6) & 248 & $(24.7)$ & 105 & $(16.7)$ & & & \\
\hline Disagree & 153 & $(9.4)$ & 91 & $(9.1)$ & 62 & $(9.8)$ & & & \\
\hline Strongly disagree & 101 & $(6.2)$ & 51 & $(5.1)$ & 50 & $(7.9)$ & & & \\
\hline \multicolumn{10}{|c|}{ People are using entertainment sources a lot } \\
\hline Strongly agree & 766 & $(46.9)$ & 432 & $(43.0)$ & 334 & $(53.0)$ & 33.99 & 4 & $<.001$ \\
\hline Agree & 449 & (27.5) & 266 & (26.5) & 183 & $(29.0)$ & & & \\
\hline Neutral & 224 & (13.7) & 160 & (15.9) & 64 & $(10.2)$ & & & \\
\hline Disagree & 106 & $(6.5)$ & 81 & $(8.1)$ & 25 & $(4.0)$ & & & \\
\hline Strongly disagree & 90 & $(5.5)$ & 66 & $(6.6)$ & 24 & (3.8) & & & \\
\hline
\end{tabular}

Couples who are going to be married soon are feeling insecure about their marriage

$\begin{array}{lllllll}\text { Strongly agree } & 706 & (43.2) & 416 & (41.4) & 290 & (46.0) \\ \text { Agree } & 418 & (25.6) & 248 & (24.7) & 170 & (27.0) \\ \text { Neutral } & 292 & (17.9) & 194 & (19.3) & 98 & (15.6) \\ \text { Disagree } & 105 & (6.4) & 70 & (7.0) & 35 & (5.6) \\ \text { Strongly disagree } & 114 & (7.0) & 77 & (7.7) & 37 & (5.9)\end{array}$

Lockdown will create an economic crisis for the country

$\begin{array}{lllllllll}\text { Strongly agree } & 1034 & (63.2) & 643 & (64.0) & 391 & (62.1) & 5.18 & 4 \\ \text { Agree } & 407 & (24.9) & 235 & (23.4) & 172 & (27.3) & & \\ \text { Neutral } & 130 & (8.0) & 88 & (8.8) & 42 & (6.7) & \\ \text { Disagree } & 20 & (1.2) & 11 & (1.1) & 9 & (1.4) \\ \text { Strongly disagree } & 44 & (2.7) & 28 & (2.8) & 16 & (2.5)\end{array}$

Pregnant women will suffer a lot for getting troubled in regular checkups

$\begin{array}{lllllllll}\text { Strongly agree } & 1009 & (61.7) & 607 & (60.4) & 402 & (63.8) & 7.81 & 4 \\ \text { Agree } & 418 & (25.6) & 252 & (25.1) & 166 & (26.3) & .099 \\ \text { Neutral } & 125 & (7.6) & 88 & (8.8) & 37 & (5.9) & (1.6) \\ \text { Disagree } & 36 & (2.2) & 26 & (2.6) & 10 & (2.4)\end{array}$

Health workers will be at high risk to work in the time of lockdown

$\begin{array}{lllllllll}\text { Strongly agree } & 1038 & (63.5) & 625 & (62.2) & 413 & (65.6) & 8.77 & 4 \\ \text { Agree } & 386 & (23.6) & 237 & (23.6) & 149 & (23.7) & .067 \\ \text { Neutral } & 133 & (8.1) & 96 & (9.6) & 37 & (5.9) \\ \text { Disagree } & 41 & (2.5) & 22 & (2.2) & 19 & (3.0) \\ \text { Strongly disagree } & 37 & (2.3) & 25 & (2.5) & 12 & (1.9)\end{array}$

Blood donation can be a serious issue because the donor can be a carrier

$\begin{array}{lllllllll}\text { Strongly agree } & 927 & (56.7) & 546 & (54.3) & 381 & (60.5) & 18.78 & 4 \\ \text { Agree } & 340 & (20.8) & 212 & (21.1) & 128 & (20.3) & & .001 \\ \text { Neutral } & 171 & (10.5) & 129 & (12.8) & 42 & (6.7) & \\ \text { Disagree } & 134 & (8.2) & 76 & (7.6) & 58 & (9.2) \\ \text { Strongly disagree } & 63 & (3.9) & 42 & (4.2) & 21 & (3.3)\end{array}$

The punishment for breaking lockdown should be very strict like in European and American countries
Strongly agree
993
(60.7)
596
(59.3)
397
(63.0) 
Table 2 Attitudes towards lockdown and gender difference of participants (Continued)

\begin{tabular}{|c|c|c|c|c|c|c|c|c|c|}
\hline \multirow[t]{2}{*}{ Characteristics } & \multicolumn{2}{|c|}{ Total $N=1635$} & \multicolumn{2}{|c|}{ Male } & \multicolumn{2}{|c|}{ Female } & \multirow[t]{2}{*}{$x^{2}$} & \multirow[t]{2}{*}{$d f$} & \multirow[t]{2}{*}{$P$-value } \\
\hline & $n$ & (\%) & $n$ & (\%) & $n$ & (\%) & & & \\
\hline Agree & 384 & $(23.5)$ & 219 & $(21.8)$ & 165 & $(26.2)$ & & & \\
\hline Neutral & 167 & $(10.2)$ & 123 & $(12.2)$ & 44 & $(7.0)$ & & & \\
\hline Disagree & 52 & $(3.2)$ & 38 & (3.8) & 14 & $(2.2)$ & & & \\
\hline Strongly disagree & 39 & $(2.4)$ & 29 & $(2.9)$ & 10 & $(1.6)$ & & & \\
\hline \multicolumn{10}{|c|}{ The government. Should increase the time of the lockdown for the safety of its people } \\
\hline Strongly agree & 889 & $(54.4)$ & 543 & $(54.0)$ & 346 & $(54.9)$ & 9.25 & 4 & .055 \\
\hline Agree & 389 & $(23.8)$ & 223 & $(22.2)$ & 166 & $(26.3)$ & & & \\
\hline Neutral & 211 & $(12.9)$ & 145 & $(14.4)$ & 66 & $(10.5)$ & & & \\
\hline Disagree & 81 & $(5.0)$ & 49 & $(4.9)$ & 32 & $(5.1)$ & & & \\
\hline Strongly disagree & 65 & $(4.0)$ & 45 & $(4.5)$ & 20 & $(3.2)$ & & & \\
\hline \multicolumn{10}{|c|}{ Online classes can be the solution to decrease the educational hindrance during lockdown } \\
\hline Strongly agree & 495 & $(30.3)$ & 284 & $(28.3)$ & 211 & $(33.5)$ & 18.61 & 4 & .001 \\
\hline Agree & 403 & $(24.6)$ & 234 & $(23.3)$ & 169 & $(26.8)$ & & & \\
\hline Neutral & 341 & (20.9) & 210 & $(20.9)$ & 131 & $(20.8)$ & & & \\
\hline Disagree & 163 & $(10.0)$ & 109 & $(10.8)$ & 54 & (8.6) & & & \\
\hline Strongly disagree & 233 & $(14.3)$ & 168 & $(16.7)$ & 65 & $(10.3)$ & & & \\
\hline
\end{tabular}

questions regarding the positive attitudes towards the lockdown (e.g., "Lockdown can prevent community spreading," see details in Table 2) with a five-point Likert scale ranging from 1 ("Strongly disagree") to 5 ("Strongly agree"), and yielding total scores ranging from 17 to 85 . An overall greater score indicates more positive attitudes towards the lockdown. The Cronbach's alpha coefficient of the attitudes towards the lockdown measures was 0.73 which indicates acceptable internal consistency of the reliability (as acceptable value $>0.6$ ) $[32,33]$.

\section{Adverse lifestyle amidst lockdown measures}

The adverse lifestyle measures were adopted from previous literature based on potential problems creating on daily life while lockdown or in home quarantine [2931]. This section included 17-item questions (7-item for positive and 10-item for negative, see details in Table 5) concerning the problems due to lockdown with three possible responses including: "Yes," "No," and "Maybe". For positive questions, the responses were coded as $0=$ Yes, 1 = Maybe, and 2 = No (e.g., "I have enough storage of food in my home"). Conversely, reverse coding as $2=$ Yes, $1=$ Maybe, and $0=$ No incorporated for negative questions (e.g., "I am smoking more than ever before"). The total score ranges from 0 to 34 , with an overall higher score indicates a greater adverse lifestyle amidst lockdown. The Cronbach's alpha coefficient of the adverse lifestyle measures was 0.64 which indicates acceptable internal consistency of the reliability (as acceptable value $>0.6)[32,33]$.

\section{Data analyses}

All statistical analyses were performed using the two analytical software (i.e., IBM SPSS Statistics version 25.0, and STATA version 13.0). Descriptive statistics (e.g., frequencies, percentages, means, standard deviations, etc.) and some first-order analyses (e.g., Chi-square tests, Fisher's exact tests, etc.) were computed. The reliability of the measures of attitudes towards lockdown and adverse lifestyle were evaluated using Cronbach alpha [32, 33]. In addition, inferential statistics including t-tests or one-way ANOVA tests were performed to determine significant relations of the mean attitudes towards lockdown and adverse lifestyle amidst lockdown scores with all examined variables. Finally, variables that significantly differed in terms of attitudes towards lockdown and adverse lifestyle amidst lockdown scores, were included in multiple regression analysis with attitudes towards lockdown and adverse lifestyle amidst lockdown measures, respectively as the independent variables. A $p$-value less than .05 was considered as significant for all statistical tests.

\section{Results}

\section{General characteristics of participants}

The samples comprised 1635 participants with their mean age 29.0 years $(\mathrm{SD}=12.2)$ and their ages ranged from 18 to 82 years. The majority of the participants were males $(61.5 \%)$, were unmarried $(61.3 \%)$, had higher secondary education (10 to 12 grades) $(63.8 \%)$, were students $(57.2 \%)$, resided in urban areas (59.0\%), and were 
Muslim (84.5\%) (Table 1). Besides, a sizeable majority resided in Dhaka division (32.5\%).

\section{Attitudes towards lockdown}

The mean scores of attitudes towards lockdown were 67.9 (SD $=8.4)$ out of 85 with an overall correct rate (positive attitudes) of $79.9 \%$. Table 2 summarized the distribution of each item attitudes-related questions along with gender differences. The participants' attitudes score was significantly higher among (i) females vs. males $(\mathrm{M}=68.9, \mathrm{SD}=7.0$ vs. $\mathrm{M}=67.3, \mathrm{SD}=9.1, p<$ $.001)$, (ii) divorced vs. unmarried $(\mathrm{M}=72.7, \mathrm{SD}=5.0$ vs. $\mathrm{M}=67.6, \mathrm{SD}=8.8, p=0.023$ ), (iii) participants having higher vs. secondary levels of education $(\mathrm{M}=71.8, \mathrm{SD}=$ 5.2 vs. $\mathrm{M}=66.4, \mathrm{SD}=8.2, p=.023$ ), (iv) housewives vs. those in business $(\mathrm{M}=69.5, \mathrm{SD}=6.5$ vs. $\mathrm{M}=64.8, \mathrm{SD}$ $=9.2, p<.001)$, (v) participants living in urban vs. rural area $(\mathrm{M}=68.2, \mathrm{SD}=8.4$ vs. $\mathrm{M}=67.4, \mathrm{SD}=8.4, p=.04)$ (Table 3).

Finally, factors that were statistically significant in the group difference analyses (t-tests/ANOVA) were included in a multiple regression analysis (see Table 4). The more positive attitudes were positively associated with the female gender, being divorced, having higher education, and students. Consequently, the residence was not significant in the multiple regression analysis. The regression model predicted $3.4 \%$ of the variance in attitudes towards lockdown $\left[F_{(13,1621)}=5.47, p<.001\right]$.

\section{Adverse lifestyle amidst lockdown}

The mean scores of adverse lifestyle amidst lockdown were $16.1(\mathrm{SD}=4.8)$ out of 34 with an overall rate of $47.4 \%$. The distribution of each item's adverse lifestylerelated question along with gender differences is presented in Table 5. The participants' adverse lifestyle

Table 3 Association between socio-demographic characteristics and mean scores of adverse lifestyle and attitudes

\begin{tabular}{|c|c|c|c|c|c|c|c|c|c|c|}
\hline \multirow[t]{2}{*}{ Characteristics } & \multicolumn{5}{|c|}{ Attitudes } & \multicolumn{5}{|c|}{ Adverse lifestyle } \\
\hline & Mean & $(S D)$ & $95 \% \mathrm{Cl}$ & $t / F$ & $p$-value & Mean & (SD) & $95 \% \mathrm{Cl}$ & $t / F$ & $p$-value \\
\hline \multicolumn{11}{|l|}{ Gender } \\
\hline Male & 67.3 & $(9.1)$ & {$[66.7,67.8]$} & 14.81 & $<.001$ & 16.6 & $(4.7)$ & {$[16.3,16.9]$} & 35.50 & $<.001$ \\
\hline Female & 68.9 & $(7.0)$ & {$[68.3,69.4]$} & & & 15.2 & $(4.8)$ & {$[14.8,15.5]$} & & \\
\hline \multicolumn{11}{|l|}{ Age } \\
\hline Young (18-25 years) & 67.8 & $(8.7)$ & {$[67.3,68.4]$} & 0.04 & .835 & 16.4 & $(4.8)$ & {$[16.1,16.7]$} & 12.69 & $<.001$ \\
\hline Older (> 25 years) & 67.9 & $(7.9)$ & {$[67.3,68.6]$} & & & 15.5 & $(4.7)$ & {$[15.1,15.9]$} & & \\
\hline \multicolumn{11}{|l|}{ Marital status } \\
\hline Unmarried & 67.6 & $(8.8)$ & {$[67.0,68.1]$} & 3.78 & .023 & 16.3 & $(4.9)$ & {$[16.0,16.6]$} & 5.03 & .007 \\
\hline Married & 68.3 & $(7.7)$ & {$[67.7,68.9]$} & & & 15.6 & $(4.7)$ & {$[15.3,16.0]$} & & \\
\hline Divorced & 72.7 & $(5.0)$ & {$[69.8,75.6]$} & & & 14.5 & $(2.9)$ & {$[12.8,16.2]$} & & \\
\hline \multicolumn{11}{|l|}{ Education } \\
\hline No formal education & 70.9 & $(5.7)$ & {$[69.4,72.4]$} & 4.53 & $<.001$ & 18.0 & $(4.0)$ & {$[16.9,19.0]$} & 6.80 & $<.001$ \\
\hline Primary & 66.5 & $(8.6)$ & {$[64.4,68.5]$} & & & 17.0 & $(4.3)$ & {$[15.9,18.0]$} & & \\
\hline Secondary & 66.4 & $(8.2)$ & {$[65.2,67.6]$} & & & 16.3 & $(4.5)$ & {$[15.7,17.0]$} & & \\
\hline Higher secondary & 67.8 & $(8.5)$ & {$[67.3,68.4]$} & & & 16.2 & $(4.8)$ & {$[15.9,16.5]$} & & \\
\hline University & 68.2 & $(8.6)$ & {$[67.2,69.3]$} & & & 14.8 & $(4.8)$ & {$[14.2,15.4]$} & & \\
\hline Higher education & 71.8 & $(5.2)$ & {$[69.9,73.6]$} & & & 14.9 & $(4.4)$ & {$[13.4,16.5]$} & & \\
\hline \multicolumn{11}{|l|}{ Occupation } \\
\hline Student & 67.9 & $(8.4)$ & {$[67.3,68.4]$} & 7.29 & $<.001$ & 16.4 & $(4.8)$ & {$[16.1,16.7]$} & 3.99 & $<.001$ \\
\hline Housewife & 69.5 & $(6.5)$ & {$[68.5,70.4]$} & & & 15.2 & $(5.1)$ & {$[14.5,16.0]$} & & \\
\hline Service holder & 68.6 & $(8.2)$ & {$[67.5,69.7]$} & & & 15.5 & $(4.8)$ & {$[14.8,16.1]$} & & \\
\hline Business & 64.8 & $(9.2)$ & {$[63.3,66.3]$} & & & 15.5 & $(4.2)$ & {$[14.8,16.2]$} & & \\
\hline Unemployed/ other & 67.9 & $(8.9)$ & {$[66.4,69.4]$} & & & 16.5 & $(4.4)$ & {$[15.8,17.3]$} & & \\
\hline \multicolumn{11}{|l|}{ Residence } \\
\hline Rural & 67.4 & $(8.4)$ & {$[66.7,68.0]$} & 4.24 & .040 & 16.8 & $(4.7)$ & {$[16.5,17.2]$} & 31.98 & $<.001$ \\
\hline Urban & 68.2 & $(8.4)$ & {$[67.7,68.8]$} & & & 15.5 & $(4.8)$ & {$[15.2,15.8]$} & & \\
\hline
\end{tabular}


Table 4 Multiple regression analysis predicting attitudes toward lockdown and adverse lifestyle

\begin{tabular}{|c|c|c|c|c|c|c|}
\hline \multirow[t]{2}{*}{ Characteristics } & \multicolumn{3}{|c|}{ Attitudes $^{a}$} & \multicolumn{3}{|c|}{ Adverse lifestyle $^{\mathbf{b}}$} \\
\hline & $B$ & $S E$ & $\beta$ & $B$ & $S E$ & $\beta$ \\
\hline \multicolumn{7}{|l|}{ Gender } \\
\hline Male & -1.06 & 0.47 & $-0.06^{*}$ & 1.52 & 0.27 & $0.15^{* * *}$ \\
\hline Female & & & c & & & c \\
\hline \multicolumn{7}{|l|}{ Age } \\
\hline Young (18-25 years) & & & & 0.70 & 0.43 & 0.07 \\
\hline Older (> 25 years) & & & & & & c \\
\hline \multicolumn{7}{|l|}{ Marital status } \\
\hline Unmarried & -5.83 & 2.30 & $-0.34^{*}$ & 1.70 & 1.30 & 0.17 \\
\hline Married & -4.11 & 2.27 & -0.24 & 1.44 & 1.30 & 0.15 \\
\hline Divorced & & & c & & & c \\
\hline \multicolumn{7}{|l|}{ Education } \\
\hline No formal education & -0.59 & 1.86 & -0.01 & 2.08 & 1.05 & $0.08^{*}$ \\
\hline Primary & -4.57 & 1.80 & $-0.11^{*}$ & 1.11 & 1.02 & 0.05 \\
\hline Secondary & -5.10 & 1.60 & $-0.19^{* *}$ & 0.82 & 0.91 & 0.05 \\
\hline Higher secondary & -3.85 & 1.55 & $-0.22^{*}$ & -0.03 & 0.89 & -0.00 \\
\hline University & -3.37 & 1.54 & $-0.15^{*}$ & -0.55 & 0.87 & -0.04 \\
\hline Higher education & & & c & & & c \\
\hline \multicolumn{7}{|l|}{ Occupation } \\
\hline Housewife & -0.49 & 1.08 & -0.02 & -0.16 & 0.63 & -0.01 \\
\hline Service holder & -0.70 & 0.98 & -0.03 & -0.11 & 0.59 & -0.01 \\
\hline Business & -4.12 & 1.06 & $-0.14^{* * *}$ & -0.97 & 0.63 & -0.06 \\
\hline Unemployed/ other & -1.84 & 1.02 & -0.06 & 0.22 & 0.60 & 0.01 \\
\hline Student & & & c & & & c \\
\hline \multicolumn{7}{|l|}{ Residence } \\
\hline Rural & -0.63 & 0.45 & -0.04 & 0.98 & 0.26 & $0.10^{* * *}$ \\
\hline Urban & & & c & & & c \\
\hline
\end{tabular}

$B$ Unstandardized regression coefficient, SE Standard error, $\beta$ Standardized regression coefficient

${ }^{*} p<.05,{ }^{* *} p<.01,{ }^{* * *} p<.001$

a Model summery (Attitudes): Covariates: Gender, Marital status, Education, Occupation and Residence; $F_{(13,1621)}=5.47, p<.001, R^{2}{ }_{\text {Adj }}=.034$

${ }^{\mathrm{b}}$ Model summery (Adverse lifestyle): Covariates: Gender, Age, Marital status, Education, Occupation and Residence; $F_{(14,1620)}=7.76, p<.001, R_{\text {Adj }}^{2}=.055$

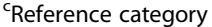

score was significantly higher among (i) males vs. females $(\mathrm{M}=16.6, \mathrm{SD}=4.7$ vs. $\mathrm{M}=15.2, \mathrm{SD}=4.8, p<$ $.001)$, (ii) young vs. older $(\mathrm{M}=16.4, \mathrm{SD}=4.8$ vs. $\mathrm{M}=$ $15.5, \mathrm{SD}=4.7, p<.001)$, (iii) unmarried vs. divorced $(\mathrm{M}$ $=16.3, \mathrm{SD}=4.9$ vs. $\mathrm{M}=14.5, \mathrm{SD}=2.9, p=.007$ ), (iv) participants having no formal education vs. higher education $(\mathrm{M}=18.0, \mathrm{SD}=4.0$ vs. $\mathrm{M}=14.9, \mathrm{SD}=4.4, p<$ $.001)$, (v) unemployed vs. housewife $(\mathrm{M}=16.5, \mathrm{SD}=4.4$ vs. $\mathrm{M}=15.2, \mathrm{SD}=5.1, p<0.001$ ), (vi) participants living in rural vs. urban area $(M=16.8, S D=4.7$ vs. $M=15.5$, $\mathrm{SD}=4.8, p<.001)($ Table 3$)$.
Finally, factors that were statistically significant in the group difference analyses ( $\mathrm{t}$-tests/ANOVA) were included in a multiple regression analysis (see Table 4). The adverse lifestyle was positively associated with male gender, having no formal education, and rural residence. Consequently, age, marital status, and occupation were not significant in the multiple regression analysis. The regression model predicted $5.5 \%$ of the variance in adverse lifestyle $\left[F_{(14,1620)}=7.76, p<.001\right]$.

\section{Discussion}

In the following study, we aimed to find out the influence on attitude and lifestyle practice due to lockdown amidst the COVID-19 pandemic among Bangladeshi residents. Lifestyles are interlinked with beliefs and attitudes, hence all of these factors play an important role in promoting health both physical and mental [34]. Since Bangladesh stands in the 18th position in terms of confirmed cases of COVID-19 across the globe as of October 24, 2020 [35], it is crucial to ensure and implement quality health promotion strategy among people to mitigate the rising physical and psychological complications during and after COVID-19.

According to our study, a desired number of participants (79.9\%) showed positive attitudes toward overcoming COVID-19 during the time of lockdown which is greater than other studies conducted in Bangladesh (62.3\%) and Pakistan (59.2\%) [5, 36]. However, an epidemiological survey accomplished in North-Central Nigeria stated that, nearly $80 \%$ participants positively supported government measures to control the pandemic in their region [1]. There were several variables among participants that showed a more positive attitude toward the pandemic in our findings. In terms of gender differences, our study showed that positive attitude was significantly higher in females $(\mathrm{M}=68.9, \mathrm{SD}=7.0)$ compared to males $(M=67.3, S D=9.1)$ which shows similarity with several studies conducted in Saudi Arabia population (female: $\mathrm{M}=28.35, \mathrm{SD}=2.32$ vs. male $\mathrm{M}=$ 28.06, SD = 3.27) [37]. Again, in terms of educational status, participants who have a higher education level $(\mathrm{M}=71.8, \mathrm{SD}=5.2)$ showed more positivity than participants having secondary level education $(\mathrm{M}=66.4, \mathrm{SD}=$ 8.2) towards attitude. The findings show similarity with a study previously conducted on Bangladeshi people during COVID-19 outbreak, which was for higher education (74.1\%) vs. secondary level education (52.2\%) [24]. Another previous study of Pakistan about knowledge, attitude, practice and risk factors regarding COVID-19, also reported slightly higher scores for post-graduation students than undergraduate students [38].

The present study also showed that housewives $(\mathrm{M}=$ $69.5, \mathrm{SD}=6.5$ ) have a higher positive attitude compared to the business occupation $(\mathrm{M}=64.8, \mathrm{SD}=9.2)$ and is 
Table 5 Adverse lifestyle and gender difference of participants

\begin{tabular}{|c|c|c|c|c|c|c|c|c|c|}
\hline \multirow[t]{2}{*}{ Characteristics } & \multicolumn{2}{|c|}{ Total $N=1635$} & \multicolumn{2}{|c|}{ Male } & \multicolumn{2}{|c|}{ Female } & \multirow[t]{2}{*}{$x^{2}$} & \multirow[t]{2}{*}{$d f$} & \multirow{2}{*}{$\begin{array}{l}P \text { - } \\
\text { value }\end{array}$} \\
\hline & $n$ & $(\%)$ & $\bar{n}$ & $(\%)$ & $\bar{n}$ & (\%) & & & \\
\hline \multicolumn{10}{|c|}{ I have enough storage of food in my home } \\
\hline Yes & 975 & $(59.6)$ & 548 & $(54.5)$ & 427 & $(67.8)$ & 28.51 & 2 & $<.001$ \\
\hline No & 439 & $(26.9)$ & 307 & $(30.5)$ & 132 & $(21.0)$ & & & \\
\hline Maybe & 221 & $(13.5)$ & 150 & $(14.9)$ & 71 & $(11.3)$ & & & \\
\hline \multicolumn{10}{|c|}{ I have sufficient medicine at home } \\
\hline Yes & 851 & $(52.0)$ & 483 & $(48.1)$ & 368 & $(58.4)$ & 16.87 & 2 & $<.001$ \\
\hline No & 593 & $(36.3)$ & 392 & (39.0) & 201 & (31.9) & & & \\
\hline Maybe & 191 & $(11.7)$ & 130 & $(12.9)$ & 61 & (9.7) & & & \\
\hline
\end{tabular}

I have maintained the lockdown properly

$\begin{array}{lllllll}\text { Yes } & 1255 & (76.8) & 741 & (73.7) & 514 & (81.6) \\ \text { No } & 189 & (11.6) & 130 & (12.9) & 59 & (9.4) \\ \text { Maybe } & 191 & (11.7) & 134 & (13.3) & 57 & (9.0)\end{array}$

$\begin{array}{llll}81.6) & 13.47 & 2 & .001\end{array}$

am saving resources for the future

$\begin{array}{lllllll}\text { Yes } & 710 & (43.4) & 421 & (41.9) & 289 & (45.9) \\ \text { No } & 735 & (45.0) & 461 & (45.9) & 274 & (43.5) \\ \text { Maybe } & 190 & (11.6) & 123 & (12.2) & 67 & (10.6)\end{array}$

I feel mental peace because of not having a workload

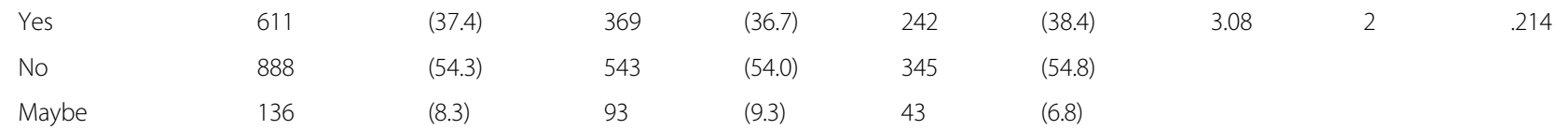

My relationship with my closest one has improved more than ever

$\begin{array}{llllllllll}\text { Yes } & 695 & (42.5) & 389 & (38.7) & 306 & (48.6) & 18.90 & 2 & <.001 \\ \text { No } & 627 & (38.3) & 424 & (42.2) & 203 & (32.2) & & & \\ \text { Maybe } & 313 & (19.1) & 192 & (19.1) & 121 & (19.2) & & \end{array}$

I use a mask when my family members gather together

\begin{tabular}{|c|c|c|c|c|c|c|c|c|}
\hline Yes & 652 & (39.9) & 378 & (37.6) & 274 & $(43.5)$ & $13.81^{\mathrm{a}}$ & 2 \\
\hline No & 956 & (58.5) & 603 & $(60.0)$ & 353 & $(56.0)$ & & \\
\hline Maybe & 27 & $(1.7)$ & 24 & (2.4) & 3 & (.5) & & \\
\hline
\end{tabular}

I very much feel bored

$\begin{array}{llllllllll}\text { Yes } & 1055 & (64.5) & 681 & (67.8) & 374 & (59.4) & 14.12 & 2 & .001 \\ \text { No } & 415 & (25.4) & 224 & (22.3) & 191 & (30.3) & & & \\ \text { Maybe } & 165 & (10.1) & 100 & (10.0) & 65 & (10.3) & & \end{array}$

I am mentally sick

$\begin{array}{lllllllll}\text { Yes } & 394 & (24.1) & 262 & (26.1) & 132 & (21.0) & 5.65 & 2 \\ \text { No } & 1123 & (68.7) & 674 & (67.1) & 449 & (71.3) & & \\ \text { Maybe } & 118 & (7.2) & 69 & (6.9) & 49 & (7.8) & & \end{array}$

I am using social media more than anything

$\begin{array}{lllllllll}\text { Yes } & 989 & (60.5) & 633 & (63.0) & 356 & (56.5) & 10.41 & 2 \\ \text { No } & 533 & (32.6) & 298 & (29.7) & 235 & (37.3) & & \\ \text { Maybe } & 113 & (6.9) & 74 & (7.4) & 39 & \text { (6.2) }\end{array}$

I am smoking more than ever before

$\begin{array}{lllllllll}\text { Yes } & 141 & (8.6) & 105 & (10.4) & 36 & (5.7) & 11.15 & 2 \\ \text { No } & 1456 & (89.1) & 876 & (87.2) & 580 & (92.1) & 004\end{array}$


Table 5 Adverse lifestyle and gender difference of participants (Continued)

\begin{tabular}{|c|c|c|c|c|c|c|c|c|c|}
\hline \multirow[t]{2}{*}{ Characteristics } & \multicolumn{2}{|c|}{ Total $N=1635$} & \multicolumn{2}{|c|}{ Male } & \multicolumn{2}{|c|}{ Female } & \multirow[t]{2}{*}{$x^{2}$} & \multirow[t]{2}{*}{$d f$} & \multirow{2}{*}{$\begin{array}{l}P \text { - } \\
\text { value }\end{array}$} \\
\hline & $n$ & (\%) & $n$ & (\%) & $n$ & (\%) & & & \\
\hline Maybe & 38 & $(2.3)$ & 24 & (2.4) & 14 & $(2.2)$ & & & \\
\hline \multicolumn{10}{|c|}{ I have found sudden changes in my sleeping pattern } \\
\hline Yes & 921 & $(56.3)$ & 570 & $(56.7)$ & 351 & $(55.7)$ & \multirow[t]{3}{*}{5.24} & \multirow[t]{3}{*}{2} & \multirow[t]{3}{*}{.073} \\
\hline No & 596 & $(36.5)$ & 374 & $(37.2)$ & 222 & $(35.2)$ & & & \\
\hline Maybe & 118 & $(7.2)$ & 61 & $(6.1)$ & 57 & (9.0) & & & \\
\hline \multicolumn{10}{|c|}{ My family members have broken the lockdown order } \\
\hline Yes & 388 & $(23.7)$ & 243 & $(24.2)$ & 145 & $(23.0)$ & \multirow[t]{3}{*}{0.32} & \multirow[t]{3}{*}{2} & \multirow[t]{3}{*}{.854} \\
\hline No & 1141 & $(69.8)$ & 698 & $(69.5)$ & 443 & (70.3) & & & \\
\hline Maybe & 106 & $(6.5)$ & 64 & (6.4) & 42 & $(6.7)$ & & & \\
\hline \multicolumn{10}{|c|}{ My productivity has decreased } \\
\hline Yes & 747 & $(45.7)$ & 473 & $(47.1)$ & 274 & $(43.5)$ & \multirow[t]{3}{*}{2.12} & \multirow[t]{3}{*}{2} & \multirow[t]{3}{*}{.346} \\
\hline No & 688 & $(42.1)$ & 410 & $(40.8)$ & 278 & $(44.1)$ & & & \\
\hline Maybe & 200 & $(12.2)$ & 122 & $(12.1)$ & 78 & (12.4) & & & \\
\hline \multicolumn{10}{|c|}{ I feel insecure about my safety } \\
\hline Yes & 962 & $(58.8)$ & 581 & $(57.8)$ & 381 & $(60.5)$ & \multirow[t]{3}{*}{1.75} & \multirow[t]{3}{*}{2} & \multirow[t]{3}{*}{.417} \\
\hline No & 580 & $(35.5)$ & 362 & $(36.0)$ & 218 & (34.6) & & & \\
\hline Maybe & 93 & $(5.7)$ & 62 & $(6.2)$ & 31 & $(4.9)$ & & & \\
\hline \multicolumn{10}{|c|}{ I easily get distracted (working slower than normal) } \\
\hline Yes & 782 & $(47.8)$ & 472 & $(47.0)$ & 310 & $(49.2)$ & \multirow[t]{3}{*}{2.87} & \multirow[t]{3}{*}{2} & \multirow[t]{3}{*}{.238} \\
\hline No & 693 & $(42.4)$ & 425 & $(42.3)$ & 268 & $(42.5)$ & & & \\
\hline Maybe & 160 & (9.8) & 108 & $(10.7)$ & 52 & (8.3) & & & \\
\hline \multicolumn{10}{|c|}{ My communication over the phone has increased } \\
\hline Yes & 1083 & $(66.2)$ & 672 & (66.9) & 411 & $(65.2)$ & \multirow[t]{3}{*}{0.78} & \multirow[t]{3}{*}{2} & \multirow[t]{3}{*}{.677} \\
\hline No & 468 & $(28.6)$ & 280 & $(27.9)$ & 188 & (29.8) & & & \\
\hline Maybe & 84 & $(5.1)$ & 53 & (5.3) & 31 & (4.9) & & & \\
\hline
\end{tabular}

${ }^{\mathrm{a} F i s h e r ' s ~ E x a c t ~ T e s t ~}$

consistent with a previously conducted study in Bangladesh [24]. In contrast, studies in Pakistan and China found that being involved in a occupation had a greater positive attitude than being unemployed or a housewife [38, 39]. However, in a multiple regression analysis, being students were associated with the more positive attitudes in the present study. In addition, according to the study finding, being divorced showed higher positivity towards COVID-19 outbreak than unmarried and it was similar according to the study conducted in Chinese residents [39].

Likewise, nearly half of the total participants (47.4\%) had adverse lifestyle scores, while the rest showed fairly good lifestyle practice. This finding is consistent with several studies conducted in India, China and Iran [40, 41]. Additionally, adverse lifestyle scores were influenced by male gender, having no formal education/lower educational status, and rural residence as per as multiple regression analysis. In agreement with this finding, males are more vulnerable compared to females during the COVID-19 pandemic [42, 43]. Accordingly, a study in Iran also reported that higher level of education provides more practice scores [44]. Several studies provide similar evidence about education plays an influential factor for healthy practice $[45,46]$. The present study found a significant association with residence factor which was seen in other studies $[47,48]$. The present study found almost $59.6 \%$ participants had sufficient storage of food and $52 \%$ had enough medication in their home during lockdown which were asked because of the scarcity of supply. It shows consistency with a study conducted in India that demonstrated almost one-third of participants were motivated to buy and stock essential commodities in case of scarcity [49]. Further, studies in Germany and Kashmir valley in India stored sufficient food for more than a week during the pandemic $[50,51]$. In addition, a sizeable minority $(42.5 \%)$ reported a better relationship with their close one during this pandemic, which 
indicates a significant risk factor for developing a chronic anxiety disorder, and depression among the rest of them [52, 53]. Indeed, quality knowledge along with positive attitude towards the COVID-19 pandemic brings good hygiene practice among people [51, 52]. Overall, appropriate knowledge during a crisis plays a key role for changing individual behavior [54].

The findings of the study predict overall increasing positive attitudes and lifestyle practice towards COVID19 during lockdown with lifestyle practice needing improvement. This study recommends that the marginalized group of people (i.e., those who don't have sufficient food or medicine at home), should be provided with necessary and consistent supports since the pandemic doesn't seem to be abated so quickly. In addition, there is also a need of promoting more quality health education program such as mass education programs both in rural and urban areas.

\section{Limitations}

The present study had several limitations. The same proportion of data could not be collected from all eight divisions of Bangladesh due to the unavailability of spontaneous participants and data collecting volunteers as the country was under complete lockdown during the study period. Also, online data collection was interrupted several times because of limited internet connectivity in some rural areas. Most of the data, including socio-demographic, medical conditions, and lockdown activities were self-reported, so there was no scope to check their authenticity. In the case of some unfortunate and vulnerable people from remote areas, they might be shown to have a negative attitude in most responses due to inaccessibility or unaffordability of available health facilities or other services. However, special attention or support should be given to them for any further research. Furthermore, the present study employed limited factors due to the unavailable information at the beginning of the COVID-19 outbreak that should be warranted in a future study with more comprehensive factors.

\section{Conclusions}

This study conveys the message about how the COVID19 lockdown has disproportionately impacted the attitudes, activities, and conditions of Bangladesh citizens across various socio-demographic conditions. The analytical outcome of this study should help to understand the response to the COVID-19 lockdown involving respondents from different niches in Bangladesh. The findings from this study should contribute to the optimization and regulation of restrictive measures depending on its necessity and effectiveness on different groups of people in the country. Overall, the study recommends a mass involvement of policymakers, health service providers, stakeholders, and general people to spread knowledge, provide necessary supports and reduce the overall risk of the pandemic like COVID-19 in Bangladesh and also in other low- and middle-income countries.

\section{Acknowledgements \\ The authors acknowledge the Community of Biotechnology (Bangladesh) and all the volunteers for their valuable contribution during the survey. The authors are also thankful to the members of Swift Integrity Computational Lab (Bangladesh) for their supports during data collection. Finally, the authors are grateful to the participants who agreed to participate in this study having confidence and trust.}

\begin{abstract}
Authors' contributions
Md. Saiful Islam: Literature searches, reviews, data curation, formal analysis, interpretation, writing draft manuscript, editing and revision of original draft. Md. Asad Ullah: Study design, data collection, formatting and revision. Ummay Soumayia Islam: Literature searches, reviews, writing draft. Sahadat Hossain: Formal analysis, interpretation, editing and revision of original draft. Yusha Araf: Conceptualization, study design and data collection. Anindya Das: Formal analysis and validation. Asir Newaz Khan: Data curation and formal analysis. Nafisa Nawal Islam: Editing and revision of original draft. Bishajit Sarkar: Data collection and validation. Abdullah Mohammad Shohael: Editing and revision of final draft and supervision. All authors approved the final version of the manuscript.
\end{abstract}

\section{Funding}

The authors received no funding from any external sources.

Availability of data and materials

All the data generated during the study are presented within the manuscript.

\section{Declarations}

Ethics approval and consent to participate

All procedures of the present study were carried out in accordance with the principle for human investigations (i.e. Helsinki Declaration). The study was approved by the Biosafety, Biosecurity, and Ethical Clearance Committee, the ethical review board of the Faculty of Biological Sciences, Jahangirnagar University (Ref. No: BBEC, JU/ M 2020(7)3). The participants were informed about the purpose of this study, and before participation in the survey, all of them provided informed informed consent. Anonymity, confidentiality, and voluntary participation with no monetary benefits were ensured, meaning that respondents could withdraw their data at any time from the study.

\section{Consent for publication}

Not applicable.

\section{Competing interests}

The authors declare no conflict of interest regarding the publication of this paper.

\section{Author details}

${ }^{1}$ Department of Public Health and Informatics, Jahangirnagar University, Savar, Dhaka, Bangladesh. ${ }^{2}$ Centre for Advanced Research Excellence in Public Health, Savar, Dhaka, Bangladesh. ${ }^{3}$ Department of Biotechnology and Genetic Engineering, Jahangirnagar University, Savar, Dhaka, Bangladesh. ${ }^{4}$ Department of Genetic Engineering and Biotechnology, Shahjalal University of Science and Technology, Sylhet, Bangladesh. ${ }^{5}$ Department of Genetic Engineering and Biotechnology, University of Dhaka, Dhaka, Bangladesh. ${ }^{6}$ Department of Economics and Social Science, BRAC University, Dhaka, Bangladesh. 
Received: 9 December 2020 Accepted: 28 September 2021

Published online: 01 November 2021

\section{References}

1. Reuben RC, Danladi MMA, Saleh DA, Ejembi PE. Knowledge, attitudes and practices towards COVID-19: an epidemiological survey in north-Central Nigeria. J Community Health. 2020;1(3):457-70. https://doi.org/10.1007/s1 0900-020-00881-1.

2. Abdelhafiz AS, Mohammed Z, Ibrahim ME, Ziady HH, Alorabi M, Ayyad M, et al. Knowledge, perceptions, and attitude of Egyptians towards the nove coronavirus disease (COVID-19). J Community Health. 2020;45(5):881-90. https://doi.org/10.1007/s10900-020-00827-7.

3. Rahman S, Bahar T. COVID-19: The New Threat. Int J Infect. 2020;7(1). https:// doi.org/10.5812/iji.102184.

4. Wang C, Horby PW, Hayden FG, Gao GF. A novel coronavirus outbreak of global health concern. Lancet. 2020;395(10223):470-3. https://doi.org/10.101 6/S0140-6736(20)30185-9.

5. Islam MS, Emran Gl, Rahman E, et al. Knowledge, attitudes and practices associated with the COVID-19 among slum dwellers resided in Dhaka City: a Bangladeshi interview-based survey. J Public Health (Bangkok). 2021;43(1): 13-25. https://doi.org/10.1093/pubmed/fdaa182.

6. Riou J, Althaus CL. Pattern of early human-to-human transmission of Wuhan 2019 novel coronavirus (2019-nCoV), December 2019 to January 2020 Eurosurveillance. 2020;25(4). https://doi.org/10.2807/1560-7917.ES.2020.25.4.2 000058.

7. Chan JFW, Yuan S, Kok KH, To KKW, Chu H, Yang J, et al. A familial cluster of pneumonia associated with the 2019 novel coronavirus indicating personto-person transmission: a study of a family cluster. Lancet. 2020;395(10223): 514-23. https://doi.org/10.1016/S0140-6736(20)30154-9.

8. Islam MS, Ferdous MZ, Islam US, Mosaddek ASM, Potenza MN, Pardhan S. Treatment, persistent symptoms, and depression in people infected with COVID-19 in Bangladesh. Int J Environ Res Public Health. 2021;18(4):1453. https://doi.org/10.3390/ijerph18041453.

9. Zhang H, Penninger JM, Li Y, Zhong N, Slutsky AS. Angiotensin-converting enzyme 2 (ACE2) as a SARS-CoV-2 receptor: molecular mechanisms and potential therapeutic target. Intensive Care Med. 2020;46(4):586-90. https:// doi.org/10.1007/s00134-020-05985-9.

10. Paul A, Sikdar D, Hossain MM, et al. Knowledge, attitudes, and practices toward the novel coronavirus among Bangladeshis: Implications for mitigation measures. PLoS One. 2020;15(9 September 2020):1-18. https:// doi.org/10.1371/journal.pone.0238492.

11. Memish ZA, Steffen R, White P, Dar O, Azhar El, Sharma A, et al. Mass gatherings medicine: public health issues arising from mass gathering religious and sporting events. Lancet. 2019;393(10185):2073-84. https://doi. org/10.1016/S0140-6736(19)30501-X.

12. Anderson RM, Heesterbeek $H$, Klinkenberg D, Hollingsworth TD. How will country-based mitigation measures influence the course of the COVID-19 epidemic? Lancet. 2020;395(10228):931-4. https://doi.org/10.1016/S0140-673 6(20)30567-5.

13. Fraser C, Riley S, Anderson RM, Ferguson NM. Factors that make an infectious disease outbreak controllable. Proc Natl Acad Sci U S A. 2004; 101(16):6146-51. https://doi.org/10.1073/pnas.0307506101.

14. Hellewell J, Abbott S, Gimma A, Bosse NI, Jarvis Cl, Russell TW, et al. Feasibility of controlling COVID-19 outbreaks by isolation of cases and contacts. Lancet Glob Heal. 2020;8(4):e488-96. https://doi.org/10.1016/ S2214-109X(20)30074-7.

15. Sakib N, Bhuiyan AKMI, Hossain S, al Mamun F, Hosen I, Abdullah AH, et al. Psychometric validation of the Bangla fear of COVID-19 scale: confirmatory factor analysis and Rasch analysis. Int J Ment Health Addict. 2020. https:// doi.org/10.1007/s11469-020-00289-x.

16. Islam MS, Tasnim R, Sujan MSH, Ferdous MZ, Sikder MT, Masud JHB, et al. Depressive symptoms associated with COVID-19 preventive practice measures, daily activities in home quarantine and suicidal behaviors: findings from a large-scale online survey in Bangladesh. BMC Psychiatry. 2021;21(1):273. https://doi.org/10.1186/s12888-021-03246-7.

17. Islam MS, Sujan MSH, Tasnim R, Sikder MT, Potenza MN, van Os J. Psychological responses during the COVID-19 outbreak among university students in Bangladesh. PLoS One. 2020;15(12):e0245083. https://doi.org/1 0.1371/journal.pone.0245083.

18. Anwar S, Araf Y, Newaz Khan A, Ullah M, Hoque N, Sarkar B, Reshad RA Islam R, Ali N, Hosen MJ. Women's Knowledge, Attitude, and Perceptions
Toward COVID-19 in Lower-Middle-Income Countries: A Representative Cross-Sectional Study in Bangladesh. Front Public Health. 2020;8:721.

19. WHO. WHO COVID-19 Situation Report:; 2020.

20. Rahman ME, Islam MS, Bishwas MS, Moonajilin MS, Gozal D. Physical inactivity and sedentary behaviors in the Bangladeshi population during the COVID-19 pandemic: an online cross-sectional survey. Heliyon. 2020;6(10): e05392. https://doi.org/10.1016/j.heliyon.2020.e05392.

21. World Health Organization. Coronavirus disease (COVID-2019) Bangladesh situation reports. https://www.who.int/bangladesh/emergencies/corona virus-disease-(covid-19)-update/coronavirus-disease-(covid-2019)-bangla desh-situation-reports. Published 2020. Accessed 30 Nov 2020.

22. Olson W. Are the Lockdown Orders Constitutional? CATO INSTITUTE. https:// www.cato.org/commentary/are-lockdown-orders-constitutional. Published 2020. Accessed 30 Nov 2020

23. Olagunju OS, Bolarinwa OA, Babalola TK. Social Distancing, Lockdown Obligatory, and Response Satisfaction During COVID-19 Pandemic: Perception of Nigerian Social Media Users. Adv J Soc Sci. 2020;7(1):44-53. https://doi.org/10.21467/ajss.7.1.44-53.

24. Ferdous MZ, Islam MS, Sikder MT, Mosaddek ASM, Zegarra-Valdivia JA, Gozal D. Knowledge, attitude, and practice regarding COVID-19 outbreak in Bangladesh: an online-based cross-sectional study. PLoS One. 2020;15(10): e0239254. https://doi.org/10.1371/journal.pone.0239254.

25. Karim A, Akter M, Mazid AT, et al. Knowledge and attitude towards COVID19 in Bangladesh: population-level estimation and a comparison of data obtained by phone and online survey methods. Mashkura Akter Mitu. 2020; 11(5):2020.05.26.20104497. https://doi.org/10.1101/2020.05.26.20104497.

26. World Health Organization. Survey tool and guidance. https://www.euro who.int/_data/assets/pdf_file/0007/436705/COVID-19-survey-tool-andguidance.pdf. Published 2020. Accessed 6 Nov 2020.

27. Centers for Disease Control and Prevention. Implementation of mitigation strategies for communities with local COVID-19 transmission. https://www. cdc.gov/coronavirus/2019-ncov/community/community-mitigation.html. Published 2020. Accessed 6 Nov 2020.

28. World Health Organization. Coronavirus disease (COVID-19) advice for the public: Mythbusters. https://www.who.int/emergencies/diseases/novelcoronavirus-2019/advice-for-public/myth-busters. Published 2020. Accessed 30 Nov 2020.

29. Khan AH, Sultana MS, Hossain S, Hasan MT, Ahmed HU, Sikder MT. The impact of COVID-19 pandemic on mental health \& wellbeing among homequarantined Bangladeshi students: a cross-sectional pilot study. J Affect Disord. 2020;277(July):121-8. https://doi.org/10.1016/j.jad.2020.07.135.

30. Mamun MA, Sakib N, Gozal D, Israfil AKM. The COVID-19 pandemic and serious psychological consequences in Bangladesh: a population-based nationwide study. J Affect Disord J. 2020;279(October):462-72. https://doi. org/10.1016/j.jad.2020.10.036.

31. Ripon RK, Mim SS, Puente AE, Hossain S, Babor MMH, Sohan SA, et al. COVID-19: psychological effects on a COVID-19 quarantined population in Bangladesh. Heliyon. 2020;6(11):e05481. https://doi.org/10.1016/j.heliyon.202 0.e05481.

32. Taber KS. The use of Cronbach's alpha when developing and reporting research instruments in science education. Res Sci Educ. 2018;48(6):1273-96. https://doi.org/10.1007/s11165-016-9602-2.

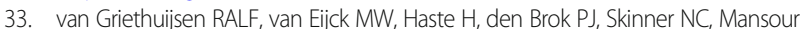
$\mathrm{N}$, et al. Global patterns in students' views of science and interest in science. Res Sci Educ. 2015;45(4):581-603. https://doi.org/10.1007/s11165-014-9438-6.

34. Mäntyselkä $P$, Kautiainen $H$, Miettola J. Beliefs and attitudes towards lifestyle change and risks in primary care - a community-based study. BMC Public Health. 2019;19(1):1049. https://doi.org/10.1186/s12889-019-7377-x.

35. Johns Hopkins University. Coronavirus Resource Center. https://coronavirus. jhu.edu/region/bangladesh. Published: 2020. Accessed 30 Nov 2020.

36. $\mathrm{Ibm} \mathrm{S}$, Ashiq $\mathrm{K}$, Sciences $P$, et al. Knowledge, attitude and practices among the inhabitants of Lahore, Pakistan towards the COVID-19 pandemic: an immediate online based cross-sectional survey while people are under the lockdown. Bangladesh J Med Sci. 2020;19:69-76.

37. Al-Hanawi MK, Angawi K, Alshareef N, et al. Knowledge, attitude and practice toward COVID-19 among the public in the Kingdom of Saudi Arabia : a cross-sectional study. Front Public Heal. 2020;8(May):1-10. https:// doi.org/10.3389/fpubh.2020.00217.

38. Afzal MS, Khan A, Qureshi UUR, Saleem S, Saqib MAN, Shabbir RMK, et al. Community-based assessment of knowledge, attitude, practices and risk factors regarding COVID-19 among Pakistanis residents during a recent 
outbreak: a cross-sectional survey. J Community Health. 2020;46(3):476-86. https://doi.org/10.1007/s10900-020-00875-z.

39. Zhong B-L, Luo W, Li H-M, Zhang QQ, Liu XG, Li WT, et al. Knowledge, attitudes, and practices towards COVID-19 among Chinese residents during the rapid rise period of the COVID-19 outbreak: a quick online crosssectional survey. Int J Biol Sci. 2020;16(10):1745-52. https://doi.org/10.7150/ ijbs.45221.

40. Singh Tomar B, Singh P, Suman S, et al. Indian community's Knowledge, Attitude \& Practice towards COVID-19. medRxiv. 2020;2020(05):05.20092122. https://doi.org/10.1101/2020.05.05.20092122.

41. Altayb Mousa KNA, Saad MMY, Tayseer Bashir Abdelghafor M. Knowledge attitudes, and practices surrounding COVID-19 among Sudan citizens during the pandemic: an online cross-sectional study. Sudan J Med Sci. 2020;15:3245. https://doi.org/10.18502/sjms.v15i5.7176.

42. Bwire GM. Coronavirus: why men are more vulnerable to Covid-19 than women? SN Compr Clin Med. 2020;2(7):1-3. https://doi.org/10.1007/s42399020-00341-w.

43. de la Vega R, Ruíz-Barquín R, Boros S, Szabo A. Could attitudes toward COVID-19 in Spain render men more vulnerable than women? Glob Public Health. 2020;15(9):1278-91. https://doi.org/10.1080/17441692.2020.1791212.

44. Erfani A, Shahriarirad R, Ranjbar K, Mirahmadizadeh A, Moghadami M. Knowledge, attitude and practice toward the novel coronavirus (COVID-19) outbreak: a population-based survey in Iran. Bull World Heal Organ. 2020; 98(3):150. https://doi.org/10.2471/BLT.20.251561.

45. Braveman PA, Cubbin C, Egerter S, Williams DR, Pamuk E. Socioeconomic disparities in health in the united States: What the patterns tell us. Am J Public Health. 2010;100(SUPPL. 1). https://doi.org/10.2105/AJPH.2009.166082.

46. Park CL, Cho D, Moore PJ. How does education lead to healthier behaviours? Testing the mediational roles of perceived control, health literacy and social support. Psychol Health. 2018;33(11):1416-29. https://doi. org/10.1080/08870446.2018.1510932.

47. Zhan S, Yang YY, Fu C. Public's early response to the novel coronavirusinfected pneumonia. Emerg Microbes Infect. 2020;9(1):534. https://doi.org/1 $0.1080 / 22221751.2020 .1732232$

48. Akalu Y, Ayelign B, Molla MD. $<p>$ knowledge, attitude and practice towards COVID-19 among chronic disease patients at Addis Zemen hospital, Northwest Ethiopia</p >. Infect Drug Resist. 2020;13:1949-60. https://doi. org/10.2147/IDR.S258736

49. Roy D, Tripathy S, Kar SK, Sharma N, Verma SK, Kaushal V. Study of knowledge, attitude, anxiety \& perceived mental healthcare need in Indian population during COVID-19 pandemic. Asian J Psychiatr. 2020;51:102083. https://doi.org/10.1016/j.ajp.2020.102083.

50. Gerhold L. COVID-19 : Risk perception and Coping strategies. Results from a survey in Germany. Interdiscip Secur Res Gr. 2020:1-11.

51. Bhat BA, Gull S, Jeelani G, et al. A study on COVID-19 lockdown impact on food, agriculture, fisheries and precautionary measures to avoid COVID-19 contamination. Galore Int J Appl Sci Humanit. 2020;4(2):8-18.

52. Teo AR, Choi H, Valenstein M. Social Relationships and Depression: Ten-Year Follow-Up from a Nationally Representative Study. PLoS One. 2013;8(4): e62396. https://doi.org/10.1371/journal.pone.0062396 Coyne J, ed.

53. Whisman MA, Uebelacker LA. Impairment and distress associated with relationship discord in a national sample of married or cohabiting adults. J Fam Psychol. 2006;20(3):369-77. https://doi.org/10.1037/0893-3200.20.3.369.

54. Glanz K, Bishop DB. The role of behavioral science theory in development and implementation of public health interventions. Annu Rev Public Health. 2010;31(1):399-418. https://doi.org/10.1146/annurev.publhealth.012809.103 604.

\section{Publisher's Note}

Springer Nature remains neutral with regard to jurisdictional claims in published maps and institutional affiliations.

Ready to submit your research? Choose BMC and benefit from:

- fast, convenient online submission

- thorough peer review by experienced researchers in your field

- rapid publication on acceptance

- support for research data, including large and complex data types

- gold Open Access which fosters wider collaboration and increased citations

- maximum visibility for your research: over $100 \mathrm{M}$ website views per year

At BMC, research is always in progress.

Learn more biomedcentral.com/submissions 